collected into tubes containing $0.5 \mathrm{ml}$ of $0.01 \mathrm{M}$ citric acid and analysed for the presence of pepsin using a lateral flow test comprising two unique human monoclonal antibodies to pepsin (Peptest ${ }^{\mathrm{TM}}$, RDBiomed Ltd). The cut off value to determine pepsin positivity was $25 \mathrm{ng} / \mathrm{ml}$.

Results Of 300 saliva samples tested, 19\% were +ve for pepsin. $64 \%$ of subjects had all three saliva samples negative; $20 \%$ had 1 sample positive, $12 \%$ had 2 samples positive and $4 \%$ had 3 samples positive. A similar percentage of samples were positive after lunch $(24 \%)$ and dinner $(22 \%)$, but lower on waking (10\%). Median acid exposure time was $0.3 \%$ (IOR $-0.1-0.8 \%$, 95 ${ }^{\text {th }}$ centile $3.5 \%$ ). Median no. of reflux events was $32(15-42,77)$ being acid $11(5-22.47)$ and non-acid 15 (8-25, 46).

Saliva samples positive for pepsin were preceded by significantly more reflux events during the 60 min interval before sampling compared to negative samples both after lunch and dinner (+ve pepsin 6 reflux (4-9) vs. -ve pepsin 3 reflux $(1-5)$ p < 0.0001). Supine acid exposure and no. of reflux episodes was not significantly different with +ve or -ve morning samples. Subjects with 3 saliva samples +ve for pepsin had a higher ratio of proximal reflux episodes than subjects with no +ve samples (37\%(range 29-40\%) vs. $19 \%(12-33 \%), p<0.02)$. Only $6 / 300$ samples contained more than $250 \mathrm{ng} / \mathrm{ml}$ pepsin.

Conclusion Pepsin was found in the expectorated saliva of a proportion of healthy individuals who did not experience reflux symptoms, particularly post-prandially. However, only $4 \%$ of healthy subjects had 3 positive samples. An increased number of reflux episodes were found prior to giving saliva samples with detectable levels of pepsin. Our results suggest that the presence of pepsin in saliva can be a potential screening tool for GERD when at least 3 samples throughout a day are positive or samples contain more than $250 \mathrm{ng} / \mathrm{ml}$ pepsin.

Disclosure of Interest None Declared

\section{PTU-150 CONCORDANCE BETWEEN ENDOSCOPIC ULTRASOUND (EUS) AND POSITRON EMISSION TOMOGRAPHY (PET) IN THE STAGING OF UPPER GASTROINTESTINAL CANCER - A DISTRICT GENERAL EXPERIENCE}

doi:10.1136/gutjnl-2013-304907.240

1."J P Seenan, 'R Sadler, ${ }^{2} \mathrm{~A} J$ S Stanley, ${ }^{1}$ S Paterson. ${ }^{1}$ Gastroenterology, Forth Valley Royal Hospital, Larbert; ${ }^{2}$ Gastroenterology, Glasgow Royal Infirmary, Glasgow, UK

Introduction Approximately 1700 patients/year are diagnosed with oesophageal or gastric cancer in Scotland. The Scottish Audit of Gastric and Oesophageal Cancer (SAGOC) previously reported under-staging of these cancers pre-operatively with curative surgery attempted too often. This resulted in incomplete resection and recurrence with a one year postoperative survival of only $53 \%$. Increasing emphasis has therefore been placed on accurate pre-operative staging. Current guidelines advise multimodal staging with CT, EUS +/- laparoscopy if gastric involvement. Recently the use of PET staging has increased. We sought to establish the concordance between EUS and PET in the staging of upper GI cancer within a large district general hospital.

Methods A retrospective study was performed in patients with oesophageal or gastric cancer referred for multimodal staging with CT, EUS and PET between October 2008 and November 2011. Each case was reviewed at the local multi-disciplinary team (MDT) meeting. MDT outcome forms were collated and a casenote review performed. Baseline demographics, tumour characteristics and TNM staging was recorded.

Results 59 patients ( 45 male) were referred for both EUS and PET. The majority had adenocarcinoma (49/59, 83.1\%) with 9 squamous carcinomas (15.3\%) and 1 carcinoid (1.7\%). A malignant stricture prevented EUS in 3 patients while in 3 patients
CT-PET revealed metastatic disease and EUS was cancelled. 53 patients (40 male) underwent staging with both modalities. Concordance of $\mathrm{N}$ staging between EUS and PET was $75.9 \%$. In $13 / 53$ patients EUS altered prior PET staging, upstaging from $\mathrm{N}_{0}$ to $\mathrm{N}_{1}$ in $12 / 13(91.4 \%)$. In patients undergoing EUS-FNA (10 mediastinal, 1 sub-diaphragmatic), 2/11 (18.2\%) patients were found to have malignant lymphadenopathy affecting PET negative nodes while in 1 patients a PET positive node was found to be benign. EUS was more accurate in predicting resection $\mathrm{N}$ stage $(65 \%)$ than PET $(38.9 \%)$ with both tending to underestimate. In patients with T3 disease there was a significant difference in $\mathrm{N}$ staging between patients undergoing resection and those treated palliatively $(p<0.05)$.

Conclusion Nodal staging by EUS and PET differs in a significant proportion of patients undergoing pre-operative work-up for upper GI cancer. In the majority of cases PET underestimates nodal staging. However, technical difficulties may preclude EUS while the finding of distant metastases at PET prior to EUS may prevent unnecessary investigations. CT and EUS remain the mainstay of pre-operative staging in oesophageal and gastric cancers but PET is a useful adjunct.

\section{Disclosure of Interest None Declared}

\section{PTU-151 HIGH RESOLUTION MANOMETRY PROFILE OF HIATAL HERNIA IN PATIENTS BEFORE AND AFTER FUNDOPLICATION}

doi:10.1136/gutjnl-2013-304907.241

1."K Bilnik, 'E Klimacka-Nawrot, ${ }^{2} \mathrm{~J}$ Kurek, 'B Blonska-Fajfrowska, ${ }^{1,2}$ A Stadnicki. ${ }^{1}$ Basic Biomedical Sciences, Medical University of Silesia, Sosnowiec; ${ }^{2}$ Department of Surgery, District Hospital, Jaworzno, Poland

Introduction Current data relating to esophageal motility evaluated by high resolution manometry(HRM) in presence of hiatal hernia $(\mathrm{HH})$ is equivocal. This study was aimed to compare HRM variables in patients with $\mathrm{HH}$ before and after fundoplication and to evaluate diagnostic performance of HRM in detecting sliding $\mathrm{HH}$.

Methods Sensitivity and specificity of HRM were assessed in 31 patients(20 females; mean age 48.2) with gastroesophageal reflux disease who were qualified for Nissen fundoplication and underwent preoperative HRM. Intraoperative diagnosis of $\mathrm{HH}$ was the gold standard. Area under curve(AUC) of receiver operating characteristic(ROC) reflecting diagnostic accuracy of HRM was also computed. Eleven patients( 5 females; mean age 52.1) out of 31 were selected who underwent both: HRM before fundoplication(preoperative group) and at least 3 months after surgery(postoperative group). Manometric protocol included 10 consecutive swallows of $10 \mathrm{ml}$ of water. Variables from pre and postoperative group were compared using paired Wilcoxon test.

Results 29 patients out of 31 were found to have HH during surgery while 14 patients had manometric criteria for $\mathrm{HH}$ (mean $\mathrm{HH}$ size was $2.44 \mathrm{~cm}$ ). Sensitivity and specificity of HRM in detecting $\mathrm{HH}$ were $48 \%$ and $100 \%$ respectively. AUC under ROC curve for HRM was 0.74 indicating limited usefulness of this method; regarding threshold value of 0.8 for clinical practise. HRM profile of $\mathrm{HH}$ in preoperative group is characterised by significantly lower minimal basal esophagogastric junction(EGJ) pressure as well as integrated relaxation pressure(IRP) comparing to postoperative group without $\mathrm{HH}$. IRP values were within normal range in both examined groups $(<15 \mathrm{mmHg}$ ). Although mean basal EGJ pressure was lower in preoperative than in postoperative group, the difference between groups didn't reach statistical significance. Neither DCI nor IBP was affected by fundoplication. Data is shown in table. 


\section{Abstract PTU-151 Table}

\begin{tabular}{lll}
\hline & $\begin{array}{l}\text { Preoperative } \\
\text { median (IOR }\end{array}$ & $\begin{array}{l}\text { Postoperative } \\
\text { median }\left(\mathbf{I O R}^{*} \text { ) }\right.\end{array}$ \\
\hline $\begin{array}{l}\text { Hiatal hernia by HRM and intraoperative } \\
\text { diagnosis }(\mathrm{n})\end{array}$ & 11 & 0 \\
Mean Basal EGJ pressure $(\mathrm{mmHg})$ & $8.3(2.6,11.2)$ & $15.8(9.9,22.8)$ \\
Minimal Basal EGJ pressure $(\mathrm{mmHg})$ & $0.5(-2.8,4.1)$ & $6.5(4.6,14.8) \dagger$ \\
IRP (mmHg) & $1.5(-0.7,3.7)$ & $5.2(2.1,11.8) \dagger \dagger$ \\
IBP (mmHg) & $14.1(9.6,18.7)$ & $13.9(7.1,24.6)$ \\
$\mathrm{DCl}(\mathrm{mmHgxsxcm})$ & $1324(711.6,2207.7)$ & $1381.7(648,2699.7)$ \\
\hline
\end{tabular}

* interquartile range; $\dagger p<0.01 ; \dagger \uparrow p<0.001$

Conclusion HRM is not reliable tool to diagnose $\mathrm{HH}$. Due to poor sensitivity of HRM in detecting $\mathrm{HH}$, manometric profile of patients with $\mathrm{HH}$ versus those without should be evaluated with caution. Surgical correction of $\mathrm{HH}$ contributes to higher EGJ relaxation pressure and improvement of antireflux barrier however neither bolus pressurisation nor DCI is affected by fundoplication.

Disclosure of Interest None Declared

\section{PTU-152 HIGH RESOLUTION MANOMETRY PATTERN OF ESOPHAGOGASTRIC JUNCTION AND ESOPHAGEAL MOTILITY IN PATIENTS BEFORE AND AFTER FUNDOPLICATION}

doi:10.1136/gutjnl-2013-304907.242

1."K Bilnik, 'E Klimacka-Nawrot, 'J Kurek, 'B Blonska-Fajfrowska, ${ }^{1,2}$ A Stadnicki. ${ }^{1}$ Basic Biomedical Sciences, Medical University of Silesia, Sosnowiec; ${ }^{2}$ Department of Surgery, District Hospital, Jaworzno, Poland

Introduction Until now it has been limited knowledge related to the application of high resolution manometry(HRM) for the evaluation of fundoplication results. The aim of this study is to assess prospectively esophagogastric junction(EGJ) relaxation and resting pressures and esophageal motility by HRM in patients with gastroesophageal reflux disease(GERD) before and after laparoscopic Nissen fundoplication.

Methods 25 patients with GERD(15 females; mean age 46.8 ) underwent HRM before(preoperative group) and at least 3 months after surgery(postoperative group). Manometric protocol included 10 consecutive swallows of $10 \mathrm{ml}$ of water. Variables from pre and postoperative group were compared using Wilcoxon test for paired samples and also McNemar's test was done to evaluate if surgery had influenced values normalisation.

Results In postoperative group mean basal EGJ pressure as well as minimal basal EGJ pressure were significantly higher than in preoperative group. Integrated relaxation pressure(IRP) was also significantly higher in postoperative group as compared with preoperative group. IRP values were within the normal range in both examined groups $(<15 \mathrm{mmHg})$ except one patient in postoperative group. Before fundoplication 11 patients had hiatal hernia, but none after surgery. Significant increase of intrabolus pressure(IBP) and decrease of contractile front velocity(CFV) were found in postoperative group as compared with preoperative group. Distal contractile integral(DCI) was significantly higher in postoperative group, however based on DCI threshold (450mmHgxsxcm) only trend from ineffective to effective esophageal motility was observed $(p=0.07)$. Also double-peaked waves were more frequent in postoperative than in preoperative group. Early dysphagia was observed in 8 of 25 patients after fundoplication. Data is shown in table.

Conclusion HRM is valuable tool for EGJ characteristics in GERD patients before and after fundoplication. Fundoplication establishes antireflux barrier by increasing EGJ resting pressures and correcting hiatal hernia. Even moderate increased of IRP may contribute to motility disorders and bolus pressurisation in some patients after fundoplication.

Disclosure of Interest None Declared

\section{PTU-153 A FORMAL SURVEILLANCE PROGRAM WITH DEDICATED ENDOSCOPY LISTS IS REQUIRED TO IMPROVE COMPLIANCE WITH THE BRITISH SOCIETY OF GASTROENTEROLOGY (BSG) GUIDELINES FOR DIAGNOSIS AND MANAGEMENT OF BARRETT'S COLUMNAR-LINED OESOPHAGUS}

doi:10.1136/gutjnl-2013-304907.243

1."K Nemeth, ${ }^{2} V$ Shah, 'A Rasheed. 'Gwent Centre for Oesophageal Diseases; ${ }^{2}$ Department of Pathology, Royal Gwent Hospital, Newport, UK

Introduction Endoscopic surveillance of Barrett's oesophagus is recommended by many national societies to detect progression to adenocarcinoma at an earlier stage.

Our aim was to audit Aneurin Bevan Health Board (ABHB) compliance with the 2005 British Society of Gastroenterology (BSG) guidelines for the diagnosis and management of Barrett's columnarlined oesophagus.

Methods Aneurin Bevan Health Board electronic prospective histopathological database was searched to identify all cases coded as Barrett's oesophagus (BO) during the period from 2005 to 2011. Endoscopy reports of all patients were matched with histology reports. A retrospective registry was then constructed including demographics, clinico-pathological features, modes and rates of follow-up, pathological progression and incident cancer rate during the study period.

Results A total of 773 cases were coded as BO during the period 2005 to 2011. Interrogation of all records confirmed 620 cases to be worthy of inclusion excluding 153 cases due to inadequate data or incorrect coding. The 620 cohort of patients consisted of 406 males and 214 females with a median age of 65 years (range 20 to 97years). $\mathrm{BO}$ histological confirmation was attained in 592/620 cases at index endoscopy and during a follow-up endoscopy in $28 / 620$ cases. Intestinal metaplasia was reported in $459 / 620$ cases. Dysplasia was diagnosed in 58/620 cases at index endoscopy; 16/620 of these index

Abstract PTU-152 Table

\begin{tabular}{llll}
\hline & Preoperative median $\left(\right.$ IOR $\left.^{*}\right)$ & Postoperative median (IOR*) & $\mathrm{p}$ value \\
\hline Mean Basal EGJ pressure $(\mathrm{mmHg})$ & $10.0(5.7-15.6)$ & $15.8(15.2-23.7)$ & $\mathrm{p}<0.05$ \\
Minimal Basal EGJ pressure $(\mathrm{mmHg})$ & $1.8(-1.1-6.5)$ & $7.3(4.6-13.9)$ & $\mathrm{p}<0.001$ \\
IRP $(\mathrm{mmHg})$ & $2.0(0-3.3)$ & $6.0(2.9-11.4)$ & $\mathrm{p}<0.001$ \\
Hiatal hernia (n, \%) & $11(45 \%)$ & 0 & \\
IBP $(\mathrm{mmHg})$ & $10.2(6.2-14.1)$ & $13.9(11.7-20.8)$ & $\mathrm{p}<0.05$ \\
DCI (mmHgxsxcm) & $859(430-1574)$ & $1008(725-1968)$ & $\mathrm{p}<0.05$ \\
CFV $(\mathrm{cm} / \mathrm{s})$ & $4.3(3.1-5.4)$ & $2.9(2.0-4.0)$ & $\mathrm{p}<0.01$ \\
Double-peaked waves $(\%)$ & $(0-22)$ & $(0-78)$ & $\mathrm{p}<0.01$ \\
\hline
\end{tabular}

* interquartile range 